\title{
MUSIKSTARS IN DER WAHRNEHMUNG JUGENDLICHER TV-CASTINGSHOW-REZIPIENTEN. EINE EMPIRISCHE UNTERSUCHUNG
}

\author{
Daniel Müllensiefen, Kai Lothwesen, \\ Laura Tiemann, Britta Matterne
}

\section{Bedingungen und Wandlungen des Starbegriffs}

Im Hinblick auf musikindustrielle Produktionsweisen ungewöhnlich ist die Offenlegung des Produktionsprozesses in den neuen TV-Castingshow-Formaten, die eine Verschiebung der Imagebewertung von Mainstream-Pop-Acts bedingt: galt das Attribut "gecastet« noch für frühe Boygroups als Stigma, scheint es nun ins Positive gewendet, als Auszeichnung derjenigen, die ein hartes Auswahlverfahren überstanden haben. Marketingstrategisch bietet sich hiermit die Möglichkeit, die anvisierte Zielgruppe auf jeder Stufe des Produktionsprozesses über die Entstehung des für sie gedachten Produktes auf dem Laufenden zu halten und so frühzeitig an das Produkt zu binden. Dieser Mechanismus eines öffentlichen Kreierens widerspricht dem tradierten Starbegriff.

In Bezug auf die TV-Castingshows als hybrides Format zwischen RealityTV und großer Fernsehunterhaltungsshow scheint es angebracht, eine deutliche Unterscheidung des Starbegriffs in zwei Konzepte vorzunehmen: Zum einen das Prinzip einer kulturindustriellen Fertigung, abgestimmt auf den Markt und bestimmt zur Verführung des Publikums; und zum anderen ein am Leistungsprinzip orientiertes Expertisemodell (vgl. Dyer 1998). In den Formaten der Castingshows werden beide Konzepte vermischt, eine eindeutige Lesart des »Stars « wird dadurch verunklart: Den Rahmen bildet das Prinzip der kulturindustriellen Fertigung, die sich in der Offenlegung des Produktionsprozesses präsentiert; das Leistungsprinzip manifestiert sich in den Darbietungen der Kandidaten, sanktioniert von den Urteilen der Juroren 
sowie in Hintergrundinformationen über die Kandidaten. ${ }^{1}$ Für die als Gegenstand unserer Teiluntersuchungen ausgewählte Show Deutschland sucht den Superstar (DSDS) gilt, was Su Holmes in ihrer Analyse des britischen Originalformats Pop Idol festgestellt hat: »Pop Idol balances elements of both manufacture and exceptionality« (Holmes 2004: 155; kursiv im Original).

Das Phänomen des Stars definiert sich zuvorderst als »soziales Konstrukt « (Sommer 1997: 114), als mehr oder minder diffuses Konglomerat von Angeboten, Vorstellungen und Bewertungen der Produktions- wie Rezeptionsseite, als deren Schnittstelle das Image fungiert. Die hierin angebotenen Lesarten können in unterschiedlicher Gewichtung in die Bedeutungszuweisungen der Fans einfließen, die mittels eigens konstruierter Sinnzusammenhänge bestimmte Facetten betonen. $^{2}$

Erfolg und Kontinuität stellen, neben dem Image, weitere Komponenten des Star-Begriffs dar (vgl. Faulstich u.a. 1997: 11). Die Integration des Stars in eine soziale Gruppe erscheint dabei als Voraussetzung, um die hervorgehobene Stellung aufgrund überdurchschnittlicher Leistungen und Eigenschaften durch direkte Interaktion zu rechtfertigen (vgl. Sommer 1997; Borgstedt/Neuhoff 2003). Diese Legitimationsbasis scheint in der Präsentation des gecasteten Stars von untergeordneter Bedeutung: Die motivierende Grundaussage der TV-Castingshows deutet an, dass potentiell jeder ein Star werden kann; das Prinzip des Willkürlichen ersetzt so in der Wahrnehmung jenes der Leistung (vgl. hierzu Neckel 2004: 9).

Im Kontext vorliegender Fan-Studien (vgl. u.a. Weyrauch 1997; Grabowski 1999; Vatterodt 2000; Rhein 2002) ist die Person des Stars als Objekt noch nicht thematisiert, der Fokus wurde vor allem auf Auswirkungen und Motivationen des Fan-Seins gerichtet. Und diese legen nahe, dass Stars als Folie Fan-eigener Projektionen fungieren und weitgehend austauschbar sind. ${ }^{3}$ Eine differenziertere Betrachtung des »Stars« wird über die Analyse

1 Zudem vermitteln die Live-Gesangsvorträge der Kandidaten eine Qualität von Authentizität, die wiederum den Gedanken an eine exzeptionelle Leistung weckt.

2 Die Frage, inwieweit auch bei Musikstars polyseme Lesarten möglich sind, wie sie bspw. für Science-Fiction-TV-Serien anhand der kreativen Produktivität der Aneignung durch die Fans dargestellt wurden (vgl. hierzu Fiske 1992; Winter 1997), ist noch weitgehend unbearbeitet; Madonna scheint für entsprechende Ansätze ein lohnendes Untersuchungsobjekt (vgl. Schmiedke-Rindt 1992). Welche typischen Dimensionen durch die mediale Vermittlung von Stars als imagetragende Kategorien festgelegt werden können, hat Silke Borgstedt (2003) am Beispiel der Berichterstattung über Robbie Williams gezeigt.

3 Dies betrifft offenbar nicht nur die zu Hochzeiten des Boygroup-Booms nach ähnlichen Schemata zusammengestellten Typen innerhalb einer Boygroup; Parallelen sind auch im Bereich der volkstümlichen Musik erkennbar (vgl. Grabowski 1999). Wichtig hingegen erscheint die Spezifizierung als Musiker (vgl. 
der von den Fans an ihre Stars herangetragenen Erwartungen und Sinngebungen möglich.

Mit der Transparenz des Produktionsprozesses und einem auf diese Art erweiterten Starbegriff (durch die Kombination der Prinzipien der industriellen Fertigung und individuellen Leistung, gepaart mit einem verschobenen Präsentationsfokus) sind wesentliche Aspekte gesetzt, unter denen die in den Castingshows produzierten »Künstler « in der Regel betrachtet werden (vgl. den Beitrag von Iris Stavenhagen in diesem Band). Wie nun nehmen die als Zielgruppe bestimmten Jugendlichen solche Produkte auf? Welche Facetten des erweiterten Starbegriffs liegen ihren Einschätzungen zugrunde? Unterscheiden sie zwischen Stars und Castingshowkandidaten? Auf welchen Kategorien fußen ihre Charakterisierungen?

An diese Bedingungen und Grundannahmen anknüpfend basieren unsere Untersuchungen auf zwei Hauptthesen, die sich auf die Einschätzung von Castingshowkandidaten durch jugendliche Rezipienten der TV-Castingshows beziehen: ${ }^{4}$

- Es werden Kategorien wahrgenommen und verhandelt, die auf spezifische Qualitäten und Leistungen verweisen, die Stars im Allgemeinen auszeichnen.

- Zudem werden Kategorien zur Einschätzung herangezogen, die auf Merkmale der Produktionsbedingungen und des Medienangebotes verweisen.

Rhein 2002: 48); entsprechend interessant wäre ein Abgleich der erforderlichen Qualifikationen, die Fans ihren Stars aus unterschiedlichen Bereichen wie z.B. Sport und Musik zusprechen.

4 Im Folgenden werden die auf dem Fragebogen versammelten Bewertungsobjekte zusammenfassend als »Musikstars« bezeichnet, wobei nicht nach dem Auftreten in bzw. der Herkunft aus TV-Castingshows unterschieden wird. Weiterhin werden die musikorientierten Fernsehcastingshows als TV-Castingshows bezeichnet, Teilnehmer von TV-Castingshows als »Castingshow-Teilnehmer «, unabhängig ihres Erfolges in den einschlägigen Formaten; als "Stars « werden Künstler bezeichnet, die außerhalb solcher Shows aufgebaut wurden (z.B. Sasha, Sabrina Setlur) oder nicht mehr als aus solchen hervorgegangen wahrgenommen werden und deren Casting-Herkunft in den Medien nicht mehr thematisiert wird (z.B. Britney Spears, Christina Aguilera, Robbie Williams, Justin Timberlake). Bei der Zusammenstellung der Objekte des Paarvergleichs im Fragebogen wurde ein ausgeglichenes Verhältnis im Bezug auf die Dimensionen Geschlecht, Nationalität und Casting-Herkunft angestrebt. 


\section{Untersuchungsmethode und Ergebnisse}

Aus diesen Thesen wurden Forschungsleitfragen entwickelt, die zur Gewinnung empirischer Daten operationalisiert wurden.

- Wird eine Differenz zwischen Castingshow-Teilnehmern und Stars wahrgenommen und spielt sie in der Beurteilung eine bedeutende Rolle?

- Nach welchen inhaltlichen Kriterien oder Dimensionen werden Castingshow-Teilnehmer beurteilt?

- Ist der durch diese Dimensionen aufgespannte Urteilsraum homogen?

- Unterscheiden sich die Beurteilungskriterien der regelmäßigen Castingshow-Rezipienten (CSR) von denen, die keine Castingshows sehen (CSI)? ${ }^{5}$

- Welches sind die Attribute, die bevorzugt zur Beurteilung herangezogen werden und über die ein Austausch von Beurteilungen stattfindet?

- Welcher Art ist der Austausch über Attribute und Beurteilungen?

- Sind die Art des Austausches und die beurteilten Attribute, die bei Wahrnehmung und Verarbeitung der TV-Castingshows durch die Rezipienten verwendet werden, durch die Art und den Inhalt der in der Show geäußerten Urteile (durch Jury und Moderatoren) beeinflusst oder vollkommen unabhängig davon?

- Wenn ein Zusammenhang zwischen den medial vermittelten Urteilen der Sendung und den Beurteilungen der Rezipienten besteht, wie stark ist er?

Da bisher keine rezipientenorientierte Studie zur Wahrnehmung und Verarbeitung von Musik-Castingshows vorliegt, sollen die Attribute und Gegenstände der hier interessierenden Beurteilungen über qualitative Methoden erfasst werden. Darüber hinaus ist auch von Interesse, in welchem Umfang und von welcher quantitativen Bedeutung die qualitativ ermittelten Beurteilungsgegenstände und Attribute sind und wie sie gegebenenfalls mit spezifischen Größen der musikalischen Lebenswelt der Rezipienten zusammenhängen.

Um der Komplexität dieses Forschungsgegenstandes Rechnung zu tragen, wurden drei methodisch und inhaltlich unterschiedliche Vorgehensweisen gewählt, die hinsichtlich der Forschungsfragen teilweise komplementäre Ergebnisse liefern sollten.

5 Im Folgenden werden aus Gründen der Redundanz die Befragten aufgrund entsprechender Äußerungen auf dem Fragebogen unterschieden in CastingshowRezipienten (CSR), als regelmäßige Zuschauer einschlägiger Sendungen, und Castingshow-Ignoranten (CSI), die entsprechende Sendungen nicht gesehen haben. 
- Mit einem umfangreichen Fragebogen wurde eine Stichprobe von Jugendlichen - durchweg Schüler - im Alter von etwa 11-17 Jahren befragt, die als wichtige Zielgruppe der TV-Castingshows gelten können. ${ }^{6}$

Durch den Fragebogen wurden sowohl Ähnlichkeitseinschätzungen zwischen bekannten Castingshow-Teilnehmern und Stars erfragt als auch Präferenzen für diese Personen mittels eines Ranking-Verfahrens. Schließlich wurden durch den Fragebogen auch soziodemographische Merkmale der Jugendlichen und Informationen über deren aktiven und passiven Umgang mit Musik erhoben. Die Auswertung dieser Ähnlichkeits- und Präferenzratings erfolgte mit den als multidimensionale Skalierung (MDS) und multidimensionale Entfaltung (MDU) bekannten quantitativen Techniken. Auch die soziodemographischen und musikspezifischen Angaben wurden quantitativ ausgewertet. Ziel war die Ermittlung des Urteilsraumes bei jugendlichen Castingshow-Zuschauern für Musikstars und Castingshow-Teilnehmer.

- Die Beiträge aus mehreren Diskussionssträngen (threads) des offiziellen Internetforums zu DSDS (http://deutschlandsuchtdensuperstar.rtl.de) wurden mittels einer an Mayring (1989) orientierten Inhaltsanalyse codiert und ausgewertet.

Die Auswertung erbrachte qualitative Erkenntnisse über das 'was < und das 'wie< des Urteilsprozesses der CSR. Da eine Vielzahl von Beiträgen unterschiedlicher Personen ausgewertet wurde, ließen sich zudem quantitative Angaben zu den verwendeten Urteilskategorien und -gegenständen machen. Ziel dieser Methode war die Präzisierung und Exemplifizierung der Urteilsvorgänge und Argumentation bei der Bewertung von Stars im Allgemeinen und Castingshow-Teilnehmern im Besonderen.

- Die in zwei Sendungen der Show DSDS geäußerten Urteile der Juroren wurden auf dieselbe Weise codiert und ausgewertet.

Auch diese Auswertung erbrachte qualitative und quantitative Erkenntnisse über Urteilsprozesse, in diesem Fall jedoch über medial vermittelte Urteile der an der Castingshow Beteiligten. Ziel war hier ein Vergleich mit den bei der Auswertung der Internetforumsbeiträge gewonnenen Ergebnissen.

6 Insgesamt wurden im März 2004 ca. 550 Fragebögen verschickt. Zu diesem Zeitpunkt näherte sich die zweite Staffel von Deutschland sucht den Superstar dem Finale und die Eindrücke der im zweiten Halbjahr 2003 zu Ende gegangenen Castingshows (Star Search, Die deutsche Stimme) konnten als noch präsent vorausgesetzt werden. Erfasst wurden die Schulformen Haupt- und Realschule, Gesamtschule sowie Gymnasium. Hinsichtlich des Alters deckten die Befragten eine weite Spanne innerhalb der Zielgruppe der jugendlichen Zuschauer ab (5. bis 11. Klasse) und auch hinsichtlich des Lebensraumes besaß die Stichprobe eine gewisse Schichtung (6 Klassen aus Großstädten, 4 Klassen aus kleinstädtischen Raum und 3 Klassen aus dem eher ländlichen Bereich). Geografisch waren Schulen aus 4 Bundesländern (Hessen, Nordrhein-Westfalen, Bremen, Hamburg) beteiligt. Die Rücklaufquote der Fragebögen lag bei ca. $60 \%$. Aufgrund fehlender Angaben erwiesen sich jedoch nur ca. 40\% der ausgesandten Fragebögen als brauchbar hinsichtlich aller Fragebogenteile. 


\subsection{Fragebogen-Daten: Soziodemographische Ergebnisse}

Im Mittel waren die befragten Schüler 13 Jahre alt. Musik spielte für die Stichprobe insgesamt eine große Rolle: Der Median der täglichen Stunden, die mit Musikhören in jeder Form verbracht werden, lag bei 5; im Mittel kauften die Schüler immerhin 1,28 und brannten 1,71 CDs im Monat. Mehr als 56\% zählten mindestens eine musikbezogene Tätigkeit zu ihren Lieblingshobbys und knapp 40\% gaben an, derzeit aktiv Musik zu machen. Die musikalischen Präferenzen verteilten sich eindeutig auf die populären Stile Pop, Rock, HipHop (Black Music) und Dance, und über 95\% gaben an, regelmäßig Musikprogramme im Fernsehen zu sehen (inkl. MTV, VIVA, Top of the Pops etc.). 68\% hatten mindestens ein Castingshow-Format regelmäßig gesehen.

Insgesamt scheint die Stichprobe also für den hier interessierenden Themenkomplex gut geeignet, sowohl hinsichtlich der Affinität der Befragten zur populären Musik an sich als auch ihrer Bekanntschaft mit durch das Fernsehen vermittelter Musik und TV-Castingshows im Speziellen.

Vergleicht man die Angaben der 68\% der Stichprobe, die mindestens ein Castingformat regelmäßig gesehen haben (CSR), mit dem restlichen Drittel der Befragten (CSI), so zeigen sich einige deutliche und sogar statistisch signifikante Unterschiede:

- Die CSR sind im Mittel signifikant jünger. Der Altersdurchschnitt liegt bei 12,8 Jahren, die CSI sind dagegen durchschnittlich 13,7 Jahre alt.

- Es befinden sich signifikant mehr Mädchen als Jungen in der Gruppe der CSR.

- Die CSR äußern verhältnismäßig mehr Präferenzen für Pop, Rock und Schlager und im Vergleich mit den CSI weniger Vorlieben für Heavy Metal, Punk und orientalischen Pop. Wenn man dies so interpretieren will, neigen die CSR eher dem musikalischen Mainstream zu.

- Von den CSR wird öfter ein Musikstar als Musik-Favorit genannt, der sich auch in der Ranking-Frage des Fragebogens befand.

Keine statistisch signifikanten Unterschiede zwischen CSR und CSI lassen sich beim Ausmaß des musikalischen Konsums und der musikalischen Aktivitäten (Kauf/Brennen von CDs, Instrumentalspiel, Singen, Kauf von Musikzeitschriften) feststellen. 


\subsection{Fragebogen-Daten: Multidimensionale Skalierung}

Im ersten Abschnitt wurden die Schüler gebeten, die Ähnlichkeit zweier Musikstars auf einer neunstufigen Skala einzuschätzen. Das Ähnlichkeitsurteil sollte möglichst spontan und >aus dem Bauch heraus erfolgen. Insgesamt waren zehn Stars vorgegeben, so dass 45 Paare beurteilt werden mussten. Von den zehn Musikstars waren fünf deutsche Castingshow-Teilnehmer und fünf etablierte Stars. In Hinblick auf die Merkmale Geschlecht und national/ international waren die Objekte ausbalanciert. Für diesen Bereich existierten zwei Varianten des Fragebogens, so dass insgesamt Ähnlichkeitspaarvergleiche zu insgesamt 20 Musikstars abgefragt wurden. ${ }^{7}$

Um die Validität der Ähnlichkeitsbewertungen zu gewährleisten, mussten die Daten eines Schülerfragebogens folgende vier Kriterien erfüllen, um in die Berechnung der Multidimensionalen Skalierung (MDS) aufgenommen zu werden: ${ }^{8}$

- Mindestens 40 der 45 Ähnlichkeitswerte müssen gültig sein, denn bei der Ersetzung von zu vielen fehlenden Werten durch Mittelwerte wird die individuelle Urteilsstruktur des Befragungsteilnehmers >verwischt<.

- Der Teilnehmer muss mindestens eine Castingshow regelmäßig gesehen haben. ${ }^{9}$

- Die Standardabweichung der neun Ähnlichkeitsratings für jeden Musikstar muss $>1$ sein, denn es ist nicht plausibel anzunehmen, dass ein Musikstar zu allen anderen Musikstars eine konstante Ähnlichkeit besitzt. ${ }^{10}$

7 Für die Ähnlichkeitsbewertungen verwendete Stars auf Fragebogenvariante A in der dortigen Reihenfolge: Alexander Klaws, Anne (von den Preluders), Britney Spears, Shaham (von Bro'Sis), Madonna, Martin Kesici (aus Star Search), Robbie Williams, Sarah Connor, Elli (DSDS 2), Xavier Naidoo; Stars der Fragebogenvariante B: Christina Aguilera, Daniel Küblböck (aus DSDS 1), Juliette (aus DSDS 1), Hila (von Bro'Sis), Justin Timberlake, Kelly Osbourne, Meiko (von Overground), Sabrina Setlur, Sasha, Vanessa (von den No Angels).

8 Insgesamt blieben nach dieser Selektion anhand der Ähnlichkeitsratings und beim jetzigen Stand der Auswertung der Fragebögen noch die Daten von 56 Vpn. übrig, die in die Berechnung der multidimensionalen Skalierung Eingang fanden, d.h. nur für diese $56 \mathrm{Vpn}$. wurde der erste Teil des Fragebogens ausgewertet.

9 In einem zweiten Schritt wäre auch denkbar, den Urteilsraum von CSR und CSI einander gegenüber zu stellen. Hier soll jedoch zunächst nur der Urteilsraum der regelmäßigen Zuschauer dargestellt werden.

10 In einem Pretest hatte sich ergeben, dass vor allem jüngere Schüler das hier geforderte Ähnlichkeitsurteil mit einem Präferenzurteil verwechseln und wenig präferierten Musikstars konstant geringe Ähnlichkeitswerte zuteilten. Dieses Antwortverhalten kann jedoch die Modellgüte einer MDS-Lösung stark beein- 
- Das Minimum der Ähnlichkeitswerte jedes Musikstars muss kleiner sein als das Maximum mindestens eines anderen Musikstars. ${ }^{11}$

Da nicht vorausgesetzt werden kann, dass sich die Urteilsräume der einzelnen jugendlichen Zuschauer hinsichtlich der Auswahl und der Gewichtung der Dimensionen gleichen, wurde der INDSCAL-Algorithmus verwendet, innerhalb dessen die Übereinstimmung der Urteilsräume geprüft werden kann. Als Distanzmaß wurde die euklidische Distanz verwendet und schließlich eine ordinale MDS gerechnet, bei der Transformationen von Distanzen der Ausgangskonfiguration zu den Disparitäten der MDS-Lösung verwendet werden, die die ordinale Information der Daten enthalten. Eine Beschränkung auf Transformationen, die auch die Intervallinformationen erhalten, wäre schon aufgrund der nur neunstufigen Rating-Skala, die eher ordinale Informationen abbildet als metrische, nicht sinnvoll gewesen. Mit der Wahl der ordinalen MDS-Variante geht eine Einschränkung des Dimensionsraumes der MDS-Lösung einher: Denn um aufgrund von ordinalen Daten zu einer intervallskalierten Lösung zu kommen, muss eine Datenverdichtung stattfinden. Nach den Empfehlungen, die Backhaus et al. (2003: 633) geben, können aus den 45 Ähnlichkeitspaaren maximal die Koordinatenwerte der zehn Objekte auf zwei bis drei Dimensionen geschätzt werden. Daraus ergibt sich, dass durch die MDS nur die Hauptdimensionen des Urteilsraumes ermittelt werden können. Es ist durchaus denkbar, dass die jugendlichen Zuschauer Castingshow-Teilnehmer und Stars insgesamt auf vier, fünf oder sechs Dimensionen einschätzen, die aber innerhalb des hier gewählten Fragebogendesigns nicht mathematisch ermittelbar sind.

Für die beiden Varianten des Fragebogens, die sich hinsichtlich der Auswahl der zehn Musikstars unterschieden, wurden zwei getrennte MDS-Lösungen berechnet. In beiden Fällen wurde aufgrund der Modellgütewerte eine dreidimensionale Lösung gerechnet. In beiden Fällen ergaben sich Werte von knapp 0,2 für das gemittelte Stress1-Maß nach Kruskal, die gemäß der Literatur noch als ausreichend für ein MDS-Modell gelten und auch in anderen entsprechenden empirischen Studien nicht selten sind. Insgesamt deuten diese nur >mittelmäßigen< Modellwerte darauf hin, dass die Versuchspersonen (Vpn.) Castingshow-Teilnehmer und Stars auf mehr als drei Dimensionen einschätzen, das Urteilsverhalten also einigermaßen differenziert ist.

trächtigen und die Interpretation einer solchen Lösung schwierig bis unmöglich machen.

11 Diese Anforderung wurde aufgenommen, um so genannte triviale oder degenerierte MDS-Lösungen auszuschließen, die dann zustande kommen können, wenn ein Beurteilungsobjekt zu allen anderen in sehr großer Unähnlichkeit steht (vgl. Borg/Groenen 1997). 
Bei der Verwendung des INDSCAL-Algorithmus ist eine Prüfung der Urteilshomogenität der urteilenden Subjekte anhand des so genannten WeirdnessIndex möglich, dessen Wertebereich von 0 bis 1 (= maximale Weirdness, d.h. Abweichung von den Urteilen der übrigen Vpn.) reicht. Keine einzige der 56 Vpn. erreichte einen Weirdness-Wert von >0,5. Das Urteil der Vpn. fiel also insgesamt recht homogen aus, so dass kein Grund besteht, einzelne Subjekte als >Ausreißer < aus der Berechnung der MDS-Konfiguration auszuschließen.

Betrachtet man die grafischen Darstellungen der beiden dreidimensionalen MDS-Konfigurationen, so lassen sich zwar keine ganz eindeutigen und unwidersprüchlichen Urteilsdimensionen herausinterpretieren, es drängen sich jedoch zur Benennung der Dimensionsachsen einige plausible Interpretationen auf. Exemplarisch seien hier die drei Dimensionen der Variante A des Fragebogens angegeben, eine ähnliche Interpretation lässt analog auch für die Variante B leisten:

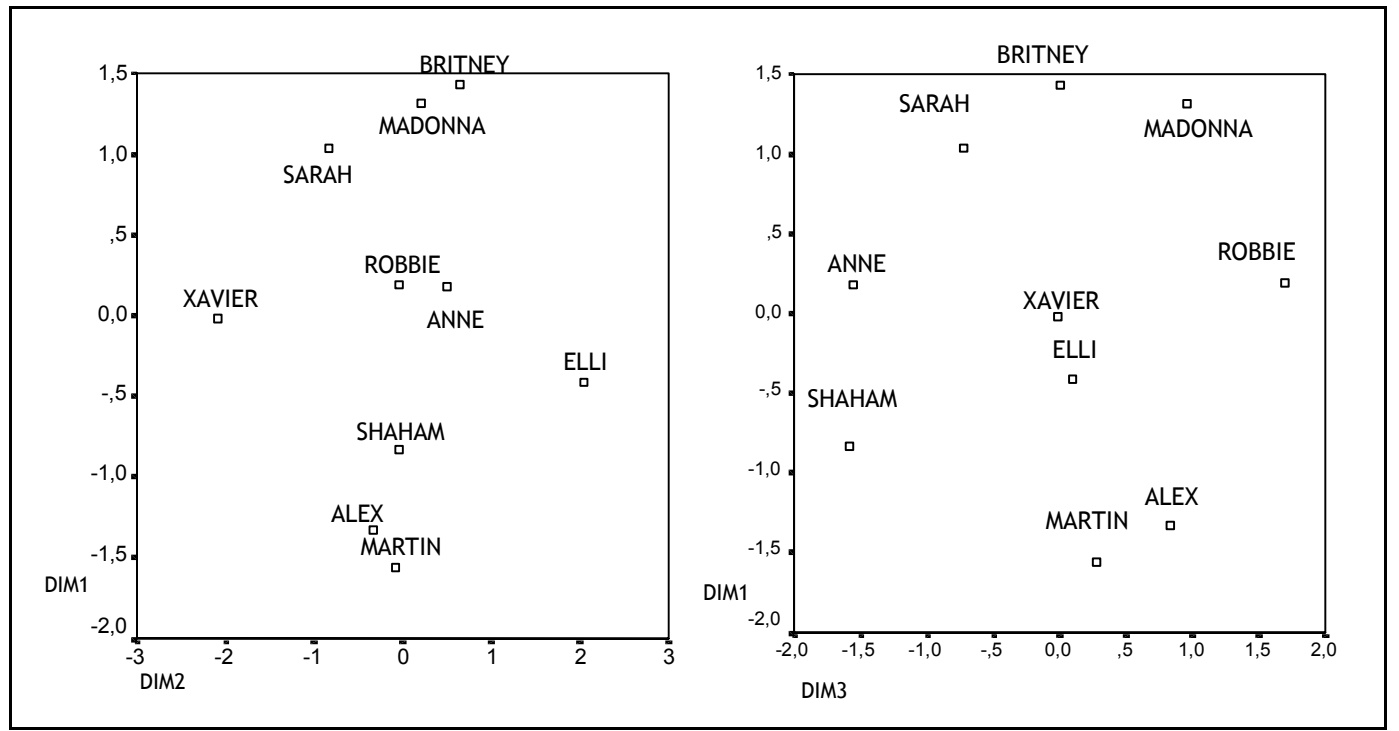

Abbildungen 1 u. 2: Dimensionen 1, 2 und 3 der MDS-Konfiguration auf Basis der Ähnlichkeitsratings der Castingshow-Rezipienten

- Dimension 1 teilt die beurteilten Stars in Castingshow-Teilnehmer (negative Werte) und Stars (positive Werte) ein. Die einzige Inkonsistenz in dieser Interpretation stellt die Castingshow-Teilnehmerin Anne (von der Girlband Preluders) dar, die einen Wert von 0,18 auf Dimension 1 besitzt. Diese Dimension wird als Kategorie Medienprodukt vs. sechter Musikstar benannt.

- Dimension 2 trennt vor allem Elli, die Gewinnerin von DSDS 2, sehr deutlich von Xavier Naidoo, während alle übrigen Objekte um den Nullpunkt 
der Dimension angeordnet sind. Diese Dimension mag für die aktuelle Medienpräsenz der Stars stehen. Elli war zum Zeitpunkt der Befragung auf dem Höhepunkt ihrer Popularität, nicht nur durch die reichweitenstarke zweite DSDS-Staffel, die zu jener Zeit auf das Finale zulief ${ }^{12}$, sondern auch durch die begleitende Berichterstattung und PR-Kampagne in zahlreichen anderen Medien (Bild-Zeitung etc.). Xavier Naidoo dagegen war zum Zeitpunkt der Befragung in den Medien so gut wie nicht präsent. Seine letzte Veröffentlichung lag fast ein ganzes Jahr zurück und war auch nur mittelmäßig erfolgreich gewesen. ${ }^{13}$ Die Bezeichnung aktuelle Medienpräsenz scheint also für die Dimension 2 plausibel.

- Für sich genommen, hält Dimension 3 auf den ersten Blick keine offensichtliche Interpretation parat. Es lässt sich jedoch an Abbildung 2 deutlich sehen, dass Dimension 1 und 3 gemeinsam die internationalen Stars (Britney, Madonna, Robbie) gut von nationalen Musikstars trennen. Alle internationalen Stars sind im rechten oberen Quadranten zu finden. Zudem liegt die sinternationale < deutsche Sängerin Sarah Connor nahe an diesem Bereich. Deshalb soll diese Dimension national vs. international benannt werden.

Es ist davon auszugehen, dass diesem jungen Publikum bei der Wahrnehmung und Beurteilung von Castingshow-Teilnehmern die Bedeutung des medialen Formates bewusst ist. ${ }^{14}$ Eine Gleichsetzung von Castingshow-Teilnehmern mit Stars, wie sie durch die mediale Präsentation im Rahmen der TVFormate und Berichterstattungen nahegelegt wird, ist in der untersuchten jungen Stichprobe nicht zu beobachten. Im Gegenteil wird die spezifische Differenz in Hinsicht auf die mediale Präsentation von den befragten Schülern mit beachtet.

12 DSDS erreichte im Jahr 2003 eine durchschnittliche Reichweite von 6,51 Mio. Zuschauern, was einem Marktanteil von $22,8 \%$ entspricht (Darschin/Gerhard 2004: 147) - wobei in diesen Angaben die letzten Sendungen der ersten Staffel mit den ersten Folgen der zweiten Staffel vermischt sind. Das Finale der zweiten Staffel erreichte am 13.3.2004 5,33 Mio. Zuschauer, was einem Marktanteil von $26,3 \%$ in der so genannten marktrelevanten Zielgruppe der Zuschauer im Alter von 14-49 Jahren entspricht (Quelle: Media Control; veröffentlicht auf www.quotenmeter.de am 14.3.2004).

13 ...Alles Gute vor uns...; VÖ-Datum: 2.6.2003; beste Platzierung in den bundesdeutschen Longplay-Charts: Platz 8 im Sommer 2003.

14 Wie diese Ansicht zeigt, lassen sich mit Sicherheit durch Drehung des Koordinatenkreuzes in der endgültigen MDS-Konfigurationen die Bereich einiger inhaltlich zusammengehöriger Objekte noch deutlicher hervorheben bzw. von den übrigen Objekten separieren. Dies wird eine Aufgabe für die Zeit nach Abschluss der Aufbereitung aller Fragebogendaten sein. 
Zusammenfassend lassen sich die Ergebnisse der explorativen Anwendung der MDS auf die durch die Schülerinnen und Schüler abgegebenen Ähnlichkeitsratings wie folgt beschreiben:

- Die Urteile der Vpn. sind recht homogen, d.h. die regelmäßigen Konsumenten der Castingsshows sind sich in ihrem Urteil relativ einig.

- Es lassen sich im Urteil der Vpn. drei Dimensionen unterscheiden, die die Art der medialen Vermittlung (Dimension: Medienprodukt vs. sechter Musikstar), das Ausmaß der Medienpräsenz (Dimension: aktuelle Medienpräsenz) und die nationale Herkunft (Dimension: national vs. international) betreffen. Dieses Ergebnis bedeutet, dass selbst die hier untersuchte sehr junge Zielgruppe bei der kognitiven Beurteilung der aus Castingshows hervorgegangenen Interpreten den medialen Entstehungskontext an vorderster Stelle mitbedenkt. Wahrscheinlich entgegen der Hoffnungen der Castingshow-Produzenten dürfte es für die im Musikmarkt agierenden Teilnehmer der Shows sehr schwer sein, die Attributierung als »gemachtes Medienphänomen« hinter sich zu lassen.

- Die nur mittelmäßigen Modellwerte der MDS-Lösung deuten darauf hin, dass die Vpn. zur Beurteilung der Musikstars noch weitere Dimensionen verwenden, die aber wegen der Beschränkung auf zehn Beurteilungsobjekte (d.h. 45 Vergleichspaare je Fragebogenversion) nicht untersucht werden können.

\subsection{Fragebogen-Daten: Multidimensionale Entfaltung}

Der zweite Teil des Fragebogens verwendete die Präferenzurteile, die die Schüler zu allen 20 aufgelisteten Musikstars abgegeben hatten. ${ }^{15}$ Die Präferenzdaten wurden mit einer der Multidimensionalen Skalierung verwandten Technik, der so genannten Multidimensionalen Entfaltung (= Multidimensional Unfolding, MDU) verarbeitet und schließlich grafisch dargestellt. Da auch die meisten Nicht-Castingshow-Seher (CSI) eine deutliche Vorstellung von den Castingshow-Teilnehmern hatten, konnten für CSR und CSI zwei getrennte MDU-Lösungen über dieselben 20 Musikstars (zehn Castingshow-

15 In diesem Teil des Fragebogens waren sowohl weniger fehlende als auch weniger formal inkorrekte Antworten vorhanden, als es für die Ähnlichkeitsvergleiche der Fall war. Offensichtlich bereitet die Vergabe von Präferenzurteilen den durchschnittlich sehr jungen Schülern sehr viel weniger Schwierigkeiten. Es wurden lediglich jene Fragebögen nicht gewertet, die 10\% oder mehr fehlende Werte enthielten bzw. bei denen eine Rangzahl mehr als fünfmal vergeben worden war. 
Teilnehmer und zehn >normale Musikstars) gerechnet und anschließend verglichen werden. ${ }^{16}$

Ergebnis beider Lösungen ist je eine vierdimensionale MDU-Konfiguration. Die Modellwerte der Konfiguration für die beiden verschiedenen Gruppen weichen deutlich von einander ab: Während sich für die CSR relativ schlechte Modellwerte ergeben (Stress 2: 0,36, RSQ: 0,877), weisen die Werte für die CSI auf eine sehr gute Modellösung hin (Stress 2: 0,0793, RSQ: 0,995). Die unterschiedliche Güte der beiden Modelllösungen lässt sich durch die einheitliche und konsequente Ablehnung fast aller CastingshowTeilnehmer durch die CSI erklären. Während die Mittelwerte aller beurteilten Musikstars bei den CSR nur zwischen 5,7 (Daniel Küblböck) und 11,5 (Christina Aguilera) schwanken, ist die Spanne bei den CSI viel größer (1,5 für Daniel Küblböck bis 14,2 für Christina Aguilera). Die Präferenz-Mittelwerte für Castingshow-Teilnehmer und Stars liegen bei den CSI deutlich und - gemäß eines t-Tests - signifikant auseinander (Mittel für CastingshowTeilnehmer: 8,57, Mittel für Stars: 12,18). Bei den CSR unterscheiden sich Castingshow-Teilnehmer und Stars nicht signifikant voneinander, was die Präferenzurteile angeht (Mittel für Castingshow-Teilnehmer: 9,57, Mittel für Stars 9,37). Regelmäßiger Konsum des Castingshow-Formates und die allgemeine Präferenz für die Teilnehmer dieser Shows hängen nachweisbar zusammen. Wenn es also die Absicht der Produzenten der CastingshowFormate war, bei den Zuschauern Präferenzen für neue Musikprodukte zu wecken, so scheint dies in gewissem Maße erfolgreich gewesen zu sein.

Auf die Darstellung der MDU-Lösungen als Grafiken soll hier aus Platzgründen verzichtet werden. Die Interpretation dieser Grafiken stützt sich wie bei der MDS - auf räumliche Nähe der beurteilten Objekte und lässt sich wie folgt zusammenfassen:

- Bei der Beurteilung der 20 Musikstars durch die CSR spielen die Dimensionen Medienprodukt vs. sechter Musikstar und national vs. international eine offensichtliche Rolle. Somit bestätigt die MDU-Lösung der CSI die Ergebnisse der MDS, die ebenfalls von den Daten der regelmäßigen Castingshow-Zuschauer ausging.

- In der MDU-Lösung auf Basis der Präferenzdaten der CSI sind, wie in den MDS-Ergebnissen, Castingshow-Teilnehmer und Musikstars voneinander unterschieden, allerdings sind hier noch das Alter und das Geschlecht der beurteilten Interpreten als Kriterien zu erkennen. Zudem liegen einige

16 Um die Urteilsdaten zu aggregieren, wurden die Präferenzratings all jener Schüler gemittelt, die denselben Lieblingsstar von der Liste der 20 Musikstars gewählt hatten, d.h. die demselben Namen den Maximalwert 20 gegeben hatten. 
Musikstars räumlich nahe beieinander, die stilistische Gemeinsamkeiten aufweisen.

- Insgesamt unterscheiden sich die Lösungen für CSR und CSI deutlich voneinander. Die regelmäßige Rezeption von Castingshow-Formaten scheint also in Beziehung zu einer bestimmten Art der Beurteilung von Musikstars zu stehen und mit einer stärkeren Beachtung der >medialen Gemachtheit der Interpreten einherzugehen.

\section{4 Äußerungen des Internetforums und der Fernseh-Jury}

Als qualitativ-quantitative Datenquelle wurden die Beiträge des offiziellen DSDS-Internetforums (http://deutschlandsuchtdensuperstar.rtl.de) sowie die Äußerungen der TV-Jury von DSDS in den Sendungen vom 13.12.2003 (»Songs aus deinem Geburtsjahr«) und 17.1.2004 (»Die Big Band-Show«) nach demselben Schema kodiert und vergleichend analysiert.

Für die Analyse der Internetforums-Beiträge wurden mehrere Diskussionsfäden (threads) ausgewählt, wobei insgesamt 580 verwertbare Aussagen entnommen werden konnten. ${ }^{17}$ Diese Beiträge schwanken im Umfang zwischen ein paar Worten und maximal ca. 20 Sätzen; Ausdruck und Wortwahl sind fast immer sehr klar und direkt, die Mitteilung erschließt sich unmittelbar. Das Forum ist nicht moderiert, der verantwortliche Betreiber (RTL) zensiert jedoch starke Kraftausdrücke und nicht jugendfreie Inhalte.

Die Jury nahm im Sendeformat DSDS eine zentrale Rolle ein. Nach jedem Auftritt eines Teilnehmers, der es unter die besten zwölf geschafft hatte, gaben die Jurymitglieder nacheinander ihre Kommentare ab. Durch Informationen zu den Tätigkeiten der einzelnen Jury-Mitglieder im Popmusikgeschäft, die zu Beginn des Formats gestreut wurden, erhielt sie aus der Perspektive des Publikums die für die Jurytätigkeit notwendige Kompetenz

17 Ein thread wird typischerweise durch eine Frage oder eine Behauptung eines Teilnehmers des Forums eröffnet. An diese Eröffnung schließen sich Kommentare oder längere argumentierende Antworten an, die sich in der Regel direkt aufeinander oder auf die eingangs gestellte Frage beziehen. Ein thread ist im Umfang nicht begrenzt und kann 50 oder mehr Beiträge enthalten. Die Beiträge werden in der Regel anonym, d.h mit einem Pseudonym unterschrieben, so dass keine soziodemografischen Angaben (Alter, beruflicher Status, Geschlecht) erkennbar sind. Zusätzlich kann eine Person auch Beiträge unter verschiedenen Pseudonymen unbemerkt ins Forum sposten<. Ausgewählt und untersucht wurden zwei einzelne größere threads und eine Zusammenstellung von mehreren kurzen threads. Der thread mit dem Thema »EIN PAAR ZEILEN ZUR SENDUNG SCHAUT REIN « wurde am 12.1.2004 begonnen; der thread »Alex ist der Beste!! « wurde begonnen am 4.1.2004, die Sammlung kurzer Beiträge stammt aus threads im Zeitraum November/Dezember 2003. 
und Legimitation zur Beurteilung junger Nachwuchssängerinnen und -sänger. Eine wichtige Aufgabe der Jury war es zu vermitteln, dass diese jungen Sängerinnen und Sänger sowie der Rahmen, in dem sie sich präsentieren, ernst zu nehmen sind. Aus den transkribierten Kommentaren der Jury in beiden Sendungen konnten 298 verwertbare Äußerungen exzerpiert werden.

Bei der Analyse der Beiträge im Internetforum ist zu beachten, dass es sich um eine besondere Textsorte handelt, vergleichbar einer Gruppendiskussion, deren Qualität nicht in der differenzierten Argumentation besteht, sondern eher in der Kommentierung von verschiedenen Aspekten der TVCastingshows und ihrer Teilnehmer, die in immer neue Zusammenhänge gestellt und mit den verschiedenen Protagonisten durchgespielt werden. Als Analyseinstrument scheinen demnach hermeneutische oder interpretative Methoden ungeeignet. Vielmehr lassen sich aus der Verteilung und Kombination von Namen, Aussagetypen, Urteilskategorien und der Wertigkeit des Urteils Erkenntnisse über die Urteilsprozesse bei den CSR und die Bedeutung der einzelnen Urteilskategorien ziehen. Diese Eigenschaften weisen auch die gesprochenen Jury-Kommentare in der Sendung auf. Insofern ist es möglich, diese beiden Textquellen mit denselben Methoden auszuwerten.

Die Stärke dieses Materials liegt in der großen Anzahl, in der Unterschiedlichkeit bzw. der vermuteten Vielzahl ihrer Autoren sowie im dialogischen Charakter: Jeder Beitrag hat konkrete Adressaten. Die gewählte Sprache und die Verdeutlichung der eigenen Position sind unmissverständlich. Das Kategorisieren und Auszählen der in den Beiträgen enthaltenen semantischen Einheiten soll diesen Besonderheiten des Textmaterials gerecht werden. Eine Orientierung hierbei bietet die von Mayring systematisch dargestellte strukturierte Inhaltsanalyse (z.B. Mayring 1989: 197f.).

Die Auswertungskategorien wurden zunächst an den Internetforumsbeiträgen entwickelt. Zentrale Fragen der Auswertung der Forenbeiträge waren:

- Nach welchen Kategorien werden die Teilnehmer der Castingshow beurteilt?

- Handelt es sich bei den Urteilen um Behauptungen, Begründungen oder Vergleiche?

- Sind die Bewertungen eher positiver oder eher negativer Art?

Die einzelnen Kategorien wurden zuvor definiert und mit entsprechenden Ankerbeispielen versehen. ${ }^{18}$ Die folgende Tabelle 1 zeigt die zur Auswertung

18 So wurde beispielsweise die Kategorie »Stimme « folgendermaßen definiert: Wohlklingende Gesangsstimme und/oder gesangliche Fähigkeiten (Intonation, Phrasierung, Rhythmus). Hierunter fielen Kommentare wie: »zu viele Töne nach 
verwendeten Urteilsgegenstände und -kategorien mit der Häufigkeit ihrer Nennungen innerhalb der ausgewählten threads des Internetforums und der Jury-Äußerungen in den beiden ausgewerteten Sendungen:

\begin{tabular}{|l|c|c|}
\hline Urteilskategorien & Internetforum & Jury \\
\hline Stimme & $166(28,7 \%)$ & $46(15,4 \%)$ \\
\hline musikalische Fähigkeit & $14(2,4 \%)$ & $15(5,0 \%)$ \\
\hline Auftritt generell & $57(9,8 \%)$ & $97(32,6 \%)$ \\
\hline Person generell & $139(24,0 \%)$ & $47(15,8 \%)$ \\
\hline Erfolg & $16(2,8 \%)$ & $5(1,7 \%)$ \\
\hline Performance/Acting & $34(5,9 \%)$ & $19(6,4 \%)$ \\
\hline Erfahrung & $5(0,9 \%)$ & $2(0,7 \%)$ \\
\hline stilistische Flexibilität & $17(2,9 \%)$ & $6(2,0 \%)$ \\
\hline Ausstrahlung/Präsenz & $25(4,3 \%)$ & $6(2,0 \%)$ \\
\hline Charakter & $52(9,0 \%)$ & $1(0,3 \%)$ \\
\hline Talent & $27(4,7 \%)$ & $2(0,7 \%)$ \\
\hline Musik/Stücke & $1(0,2 \%)$ & $26(8,7 \%)$ \\
\hline eigene Musikerpersönlichkeit & $4(0,7 \%)$ & $3(1,0 \%)$ \\
\hline Aussehen & $18(3,1 \%)$ & $10(3,4 \%)$ \\
\hline Styling & $4(0,7 \%)$ & $13(4,4 \%)$ \\
\hline Total & $579(100 \%)$ & $298(100 \%)$ \\
\hline
\end{tabular}

Tabelle 1: Kategorien der im DSDS-Internetforum und von der TV-Jury geäußerten Urteile mit zugehörigen Häufigkeiten (Anzahl der Nennungen; Prozentangaben in Klammern)

Mit Abstand am häufigsten werden im Internetforum die Stimmen der Teilnehmer beurteilt, was für einen Gesangswettbewerb nicht als ungewöhnlich gelten kann. Danach folgen pauschale Urteile über die Person im Allgemeinen, in denen nicht weiter spezifiziert ist, was an einem Teilnehmer gut oder schlecht ist. Es schließen hieran Urteile über einzelne Aspekte der Castingshow-Teilnehmer an, wie z.B. die Auftritte in der Show, den vermeintlichen Charakter der Kandidaten oder die Darstellung auf der Bühne. Insgesamt tauschen sich die Teilnehmer des Forums sowohl über musikalische (Stimme, musikalische Fähigkeiten, stilistische Flexibilität) wie auch über außer-musikalische Aspekte (Charakter, Ausstrahlung, Aussehen) aus.

oben oder unten weg « oder »gesanglich immer schlecht«. Eine weitere häufig genannte Kategorie war der »Auftritt generell«. Definition: Einzelne Auftritte ohne Spezifizierung des Bewerteten. Beispiele hierfür waren: »gute Arbeit geleistet« oder »letzter Auftritt war wirklich sehr gut gewesen«. 
Um Zusammenhang und Übereinstimmungen zwischen den Häufigkeiten der verwendeten Urteilskategorien im Forum und durch die Jury zu ermitteln, wurde eine Kontingenzanalyse durchgeführt, die Aufschluss über den Zusammenhang von Häufigkeitsverteilungen in nominal skalierten Variablen gibt. Ein $\chi^{2}$-Test zeigte auf dem Signifikanzniveau von 0,01 an, dass signifikante Unterschiede zwischen den Häufigkeitsverteilungen in den beurteilten Aspekten zwischen Jury und Forum bestehen. Im Vergleich zu den Äußerungen im Internetforum beurteilte die Jury weit weniger häufig die Stimme und die Person generell (15,4\% bzw. 15,8\% aller Kommentare), den Auftritt der Kandidaten im Allgemeinen aber deutlich häufiger (32,6\%). Daraus lässt sich folgern, dass für die Partizipanten des Internetforums die Stimme und die persönlichen Eigenschaften der Castingshow-Teilnehmer im Mittelpunkt der Betrachtung standen. Im Internetforum wurde DSDS hauptsächlich als -Gesangswettbewerb < wahrgenommen, obwohl die Juroren ein stärkeres Gewicht auf die Performance der Teilnehmer auf der Studiobühne legen. Um die Stärke des Zusammenhangs zwischen den beiden Häufigkeitsverteilungen zu ermitteln - der trotz der signifikanten Unterschiede bestehen mag -, wurde ein Vergleich der Urteilshäufigkeiten mit Cramers V-Koeffizient durchgeführt, der einen Wert von 0,455 ergab. Die von Jury und Internetschreibern beurteilten Kategorien sind also nicht vollkommen unabhängig voneinander: In der Auswahl der wichtigsten Urteilskategorien scheinen die Rezipienten dem Beispiel der Jury zu folgen.

Betrachtet man die Form der Äußerungen im Internetforum und unter den Jury-Beiträgen sowie ihre jeweiligen Häufigkeiten in der folgenden Tabelle, so fällt die überwiegende Zahl der Behauptungen auf, also Aussagen, die keine weitere Erklärung formulieren (88,1\% bzw. 86\%).

\begin{tabular}{|l|l|l|l|l|}
\hline & Behauptungen & Begründungen & Vergleich & Total \\
\hline Internetforum & $510(88,1 \%)$ & $24(4,1 \%)$ & $45(7,8 \%)$ & $579(100 \%)$ \\
\hline Jury & $257(86,0 \%)$ & $7(2,3 \%)$ & $35(11,7 \%)$ & $299(100 \%)$ \\
\hline
\end{tabular}

Tabelle 2: Äußerungsformen der im DSDS-Internetforum und von der TV-Jury geäußerten Urteile mit zugehörigen Häufigkeiten

Begründungen werden so gut wie nicht geliefert und auch Vergleiche (z.B. mit anderen Musikern oder zwischen den Teilnehmern) sind eher selten. Dies mag durch die Schriftform im Medium Internet forciert sein, bei der kurze Äußerungen präferiert werden und komplexere und längere Äußerungen eher Gefahr laufen, ignoriert zu werden. Für die mündlichen Äußerungen der Jury kann die Begründung allerdings nicht herhalten. Hier scheint 
eher eine Fokussierung auf kurze und plakative Statements für die Form der Äußerungen verantwortlich zu sein.

Ein $\chi^{2}$-Test zeigte keine signifikanten Differenzen zwischen der Art der Äußerungen von Internetforumsteilnehmern und der Juroren an: Auch die vermeintlich kompetente Jury macht im selben geringen Maße von Begründungen und Vergleichen in ihren Urteilen Gebrauch.

Als letztes wurde die Valenz der Äußerungen im Forum auf einer PositivNegativ-Skala beobachtet. Im Vorfeld der Analyse wurden Kriterien für die Zuweisung der in den Aussagen verwendeten Attribute zu den fünf Stufen der Valenz-Skala festgelegt. Im Ergebnis zeigt sich ein annäherndes Gleichgewicht zwischen positiven und negativen Aussagen unter den Internetbeiträgen sowie ein deutliches Übergewicht positiver Aussagen von der Jury, wie die folgende Tabelle erkennen lässt.

\begin{tabular}{|l|l|l|r|r|l|l|}
\hline & $\begin{array}{l}\text { sehr } \\
\text { positiv }\end{array}$ & positiv & indifferent & negativ & $\begin{array}{l}\text { sehr } \\
\text { negativ }\end{array}$ & Total \\
\hline Forum & $12(2,1 \%)$ & $226(39,0 \%)$ & $132(22,8 \%)$ & $188(32,4 \%)$ & $22(3,8 \%)$ & $580(100 \%)$ \\
\hline Jury & $55(18,4 \%)$ & $123(41,1 \%)$ & $51(17,1 \%)$ & $61(20,4 \%)$ & $9(3,0 \%)$ & $299(100 \%)$ \\
\hline
\end{tabular}

Tabelle 3: Wertungen der im DSDS-Internetforum und von der TV-Jury geäußerten Urteile mit zugehörigen Häufigkeiten

Entsprechend zeigte ein $\chi^{2}$-Test für die Positiv-Negativ-Einstufungen signifikante Unterschiede zwischen Forum und Jury. Ein Wert von 0,307 für das Zusammenhangsmaß Cramers $\mathrm{V}$ bedeutet einen mäßigen Zusammenhang zwischen den von Jury und Forum verwendeten Valenzen. Im Forum meldeten sich eben nicht nur Fans, sondern auch viele Kritiker des Sendeformats Castingshow, insbesondere von DSDS und der einzelnen Interpreten zu Wort. Diese Auszählung bestätigt dennoch den Eindruck, dass das DSDS-Forum nicht vorrangig ein >Lästerforum< ist, sondern dass sich dort durchaus auch die Fans einzelner Teilnehmer und regelmäßige Rezipienten des TV-Formates finden. Dies mag auch ein Grund für die Fülle der Beiträge und die rege Beteiligung an diesem Forum sein.

Die Jury erfüllte dagegen ihre eingangs vermutete Funktion, die Kandidaten und damit das Sendeformat, durch den inflationären Einsatz lobender Äußerungen aufzuwerten. 


\section{Diskussion}

Die Ergebnisse unserer Untersuchungen sind gemäß den eingangs formulierten Thesen in zwei Bereiche zu fassen. Zum einen treten Effekte hervor, die auf die genutzten Medien, die TV-Sendungen und das Internetdiskussionsforum, zurückzuführen sind. Zum anderen, und teilweise an die erstgenannten anschließend, sind die Beziehungen zwischen den TV-Rezipienten und den Castingshow-Teilnehmer wie auch den Stars zu betrachten.

\subsection{Medieneffekte}

Die Wirkung medial vermittelter Inhalte verläuft nicht eindimensional, sondern kann durch mannigfache Möglichkeiten der Intervention seitens der Rezipienten beeinflusst werden. Nach der Agenda Setting-Theorie (Jäckel 1999: 158ff.) vollzieht sich dabei Medienwirkung auf unterschiedlichen Stufen. ${ }^{19}$ So wird zunächst eine Aufmerksamkeit für bestimmte Themen erreicht (»awareness of the issues«), denen eine Anhäufung von Informationen folgt (»body of information«). Die Auswahl aus diesem Informationsangebot führt zur Ausbildung von Einstellungen und Urteilen (»attitude formation«), die schließlich Verhaltensänderungen bewirken können (»attitudes shape behaviour «) (Lowery/DeFleur 1995: 275, zit. n. Jäckel 1999: 164). Daran anschließend gehen wir davon aus, dass jugendliche TV-Rezipienten aus den in den einschlägigen TV-Castingshows präsentierten Informationen auswählen, ihre Einstellungen und Urteile danach ausbilden und entsprechend handeln, was sich im Antwortverhalten auf den Fragebogen ebenso niederschlägt wie in den Beiträgen im Internetdiskussionsforum.

In den untersuchten Diskussionsfäden äußert sich ein durchaus vorhandenes Bewusstsein für den medialen Kontext, das die Sendeformate und deren Protagonisten (Kandidaten, Jury, Moderatoren) wie auch die Produktionsbedingungen und die stilistische Ausrichtung der musikalischen Produkte berührt. Auch sind Einwirkungen jeweils aktueller, in den Medien vertretener Inhalte aufzuzeigen. (So legt eine MDS-Lösung eine Einschätzung der Kandidaten und Stars anhand aktueller musikalischer Veröffentlichungen nahe.)

In der Art der Auseinandersetzung mit den angesprochenen Themen, der Diskussion und Argumentation im Internetforum zeigen sich Parallelen zu dem im DSDS-Format präsentierten Wertungs-Schema. Hier kann von einer

19 Zur Bedeutung der Agenda Setting-Theorie für die musikalische Urteilsbildung vgl. Behne 1997 und Rösing/Barber-Kersovan 1998. 
Übernahme des Jury-Modells gesprochen werden. Dieses bezeichnet in idealtypischer Weise eine nicht-argumentative Auseinandersetzung, geprägt durch subjektive Geschmacksurteile, die nicht näher erörtert oder begründet werden. Die offenbar differenziertere Urteilsfähigkeit der untersuchten CSR- gegenüber den CSI-Gruppen ist in gleicher Weise zu interpretieren wie die Diskussion musikalischer Inhalte (Jury-Urteile, Kompetenz der Juroren) im Internetforum: Über die in den TV-Formaten vermittelten musikalischen Inhalte wird Wissen akkumuliert, das zum Ausbau der Urteilsbasis dient. So gesehen vollzieht sich über die Rezeption von Castingshows auch eine Aneignung von Kompetenzen in Bezug auf musikalische Aspekte, die wiederum in die Beiträge des Internetforums einfließen können. Vor dem Hintergrund des Ansatzes der Cultural Studies betont Rainer Winter (1997: 49f.) die Produktivität der Fans bei der Aneignung medialer Inhalte (»Texte«) und unterscheidet aufgrund der Analyse von Fantexten (Fanzines, Bücher, Videos) verschiedene Arten kreativen Umgangs. Das für den vorliegenden Fall der TV-Castingshows interessante Verhalten ist die von Winter so benannte »wissenschaftliche Analyse «: »Die Kreativität in diesem Prozeß liegt in der Systematisierung des textuellen Materials und im Vergleich, in der Analyse und in der Konstruktion neuer Verbindungen im Netz der Intertextualität « (Winter 1997: 50). Indem Castingshow-Teilnehmer untereinander wie auch an Stars gemessen werden, Bezüge der Urteile und Einschätzungen zu Juroren wie auch zu anderen Stars aufzuzeigen sind, erzeugen die Rezipienten ein eigenes System, das auf den oben dargestellten Urteilskategorien fußt.

\subsection{Der Star aus Sicht der Fans}

Beim Aufbau von Fan-Star-Beziehungen bietet sich das Konzept der parasozialen Beziehung (PSB) als Interpretationsmodell an. Dieses medientheoretische Konzept zielt auf die Aktivierung des Publikums über eine mögliche Abstufung des /Involvement der TV-Rezipienten und »bezieht sich auf die wahrgenommenen affektiven oder emotionalen Beziehungen von Mitgliedern des Publikums mit Medienpersönlichkeiten « (Rubin 2000: 146). Diese können ähnliche Verhaltensweisen und Reaktionen bedingen wie in »realen sozialen Interaktionen und Beziehungen«. Darüber hinaus zeigt sich eine Stabilität und Kontinuität jener Beziehungen »auch außerhalb der Rezeptionssituation [...] und auch in Abwesenheit des parasozialen >Beziehungspartners « (Six/ Gleich 2000: 363). ${ }^{20}$ Insofern können die in den Ergebnissen unserer Untersuchungen festgestellten Qualitäten als Einstellungen vermutet werden, die

20 Vgl. hierzu die Ergebnisse einschlägiger Studien zum Fan-Verhalten von Boygroup-Anhängern bei Vatterodt 2000 und Hauk 1999. 
auch zwischen den Ausstrahlungen der einzelnen Sendungen andauern. Zudem konnten in der Auswertung der Fragebogen-Urteile Unterschiede in der Einschätzung von Castingshow-Teilnehmer und Stars ausgemacht werden, die auf eine Unterscheidung von Solokünstlern und Band-Mitgliedern hinweisen. Für den Aufbau einer PSB kann dies bedeuten, dass die angebotenen Ansatzpunkte hinsichtlich charakteristischer Attribute differieren, je nachdem ob konkrete Personen vermittelt werden oder deren Einbindung in einen Gruppen- oder Bandkontext gegeben ist. So könnte bei einem BandMitglied eher das Outfit im Vordergrund stehen als die Leistungsfähigkeit einer ausgebildeten Stimme. Am anschaulichen Beispiel Daniel Küblböcks wäre in diesem Kontext nachzuspüren, ob und wie sich die Präsenz in den Medien als Basis für eine zeitlich länger andauernde PSB auswirken kann.

Die differenzierte Betrachtung der Objekte durch die TV-Rezipienten spricht für eine Fokussierung des Interesses auf musikalische Qualitäten und charakteristische Eigenschaften und Eigenheiten der Stars, die im Internetforum diskutiert werden. Ähnliches konnte auch Stefanie Rhein in ihrer Studie zur Differenzierung des Fantums ${ }^{21}$ feststellen:

»Während die musikbezogene Dimension für die Fans die wichtigste Rolle spielt, sind die Items der starbezogenen Dimensionen (z.B. Schwärmen) für sie weit weniger bedeutsam. Die Annahme, dass den Teenie-Fans der Star wichtiger sei als die Musik, wird durch diese Ergebnisse widerlegt: Die befragten Fans sehen in der Begeisterung für die Musik die zentrale Bedeutung ihres Fans-Seins« (Rhein 2002: 48).

Die Bedeutung von kommunikativen Prozessen ist in diesem Zusammenhang nicht zu unterschätzen. ${ }^{22}$ In den Internetdiskussionsforen scheinen solche Prozesse durch die in den Jury-Urteilen angebotenen Kategorien angeleitet zu werden. Auf diese Weise sind die Ergebnisse der vorgestellten Teilstudien zusammenzufassen: Den Kandidaten werden Attribute zugewiesen, die sie als Stars qualifizieren. Dennoch erscheinen sie in der Einschätzung durch die Rezipienten von ihnen abgesetzt.

21 Rhein (2002) legte 217 Vpn. im Alter von 11 bis 15 Jahren einen Multimedia-Fragebogen vor; Ergebnis der Studie ist eine Typologie »unterschiedlicher Intensitäten« des Fantums.

22 Gerade im Hinblick auf den Aufbau einer Fangemeinde als Interessensgruppe sowie vor dem Hintergrund kultursoziologischer Interpretationen, die auf das Verhältnis von Kulturindustrie und (möglicher) Selbstbestimmung des Publikums zielen (vgl. hierzu Fiske 1992; Winter 1997). 


\subsection{Weiterführung}

Im Anschluss an die vorgestellten Ergebnisse unserer Studie eröffnen sich weitere Fragestellungen. So wären die aufgezeigten Zusammenhänge zwischen Juroren- und Fan-Urteilen im Hinblick auf die Konstitution von FanGruppen zu vertiefen; darüber hinaus könnten zudem Einblicke in die allgemeinen kommunikativen Prozesse und Strukturen von Fangemeinden gewonnen werden, wie auch deren Nutzung neuer Kommunikationstechnologien. Gerade aber die Bevorzugung von »echten« Musikstars gegenüber den aus Castingshows hervorgegangenen Künstlern, wie sie in den Fragebögen geäußert wird, bietet Ansatzpunkte, die Produktionsstrategien der Musikindustrie zu analysieren und die Akzeptanz dieser Produkte zu erfragen. Hier bestünde eine Möglichkeit der Rückkopplung an kommunikationstheoretische Ansätze wie der Agenda Setting-Theorie, die den Rahmen einer Längsschnittstudie abstecken und auf diese Weise Interessen der Rezipienten- und das Angebot der Produktionsseite abgleichen könnte. Eine solche Studie wäre in der Lage, das Phänomen der industriell gefertigten jugendorientierten Mainstream-Popmusik seit den Erfolgen der ersten Boygroups bis zu den diversen TV-Casting-Formaten zu umspannen. Erste methodische Ansätze und Ergebnisse dazu haben wir in unseren Teiluntersuchungen darzulegen versucht.

\section{Literatur}

Backhaus, Klaus et al. (2001). Multivariate Analysemethoden. Berlin: Springer (10. Aufl.).

Behne, Klaus Ernst (1997). »Musikästhetik. III. Musikalische Urteilsbildung.«In: Die Musik in Geschichte und Gegenwart. Sachteil, Bd. 9. Hg. v. Ludwig Finscher. Kassel u.a.: Bärenreiter/Metzler (2. Aufl.), Sp. 998-1012.

Borg, Ingwer/Groenen, Patrick (1997). Modern Multidimensional Scaling. Theory and Applications. Berlin: Springer.

Borgstedt, Silke (2004). »Das inszenierte Erfolgsmodell. Robbie Williams im Spiegel der Tagespresse.« In: Samples 3, S. 9-18 (http://www.aspm-samples.de/ Samples3/borgstedt.html; Zugriff: 10.7.2004).

Borgstedt, Silke/Neuhoff, Hans (2003). »Der Kapitalismus ist Sieger.«In: Rheinischer Merkur, Nr. 30 vom 24. Juli.

Darschin, Wolfgang/Gerhard, Heinz (2004). »Tendenzen im Zuschauerverhalten. Fernsehgewohnheiten und Fernsehreichweiten im Jahre 2003.«In: Media Perspektiven, H. 4, S. 142-150.

Dyer, Richard (1998). Stars. London: British Film Institute (Neuaufl.).

Faulstich, Werner/Korte, Hermann/Lowry, Stephen/Strobel, Ricarda (1997). » Kontinuität - - zur Imagefundierung des Film- und Fernsehstars. «In: Der Star. 
Geschichte - Rezeption - Bedeutung. Hg. v. Werner Faulstich und Helmut Korte. München: Fink, S. 11-28.

Fiske, John (1992). »The Cultural Economy of Fandom. «In: The Adoring Audience. Fan Culture and Popular Media. Hg. v. Lisa A. Lewis. London, New York: Routledge, S. 30-49.

Grabowski, Ralf (1999). »Zünftig, bunt und heiter.«Beobachtungen über Fans des volkstümlichen Schlagers. Tübingen: Tübinger Vereinigung für Volkskunde.

Hauk, John (1999). Boygroups! Teenager, Tränen, Träume. Mit einem Lexikon der Boygroups der Musikgeschichte und einer Befragung von über 500 Fans der aktuellen Boygroups. Berlin: Schwarzkopf und Schwarzkopf.

Holmes, Su (2004). » Reality Goes Pop!< Reality TV, Popular Music, and Narratives of Stardom in Pop Idol.« In: Television \& New Media 5, Nr. 2 (Mai), S. 147-172.

Jacke, Christoph (1998). »Millionenschwere Verweigerer: Die US-Rockband Nirvana.« In: Neues zum Umgang mit Rock- und Popmusik. Hg. v. Helmut Rösing und Thomas Phleps (= Beiträge zur Popularmusikforschung 23). Karben: Coda, S. 7-30.

Jacke, Christoph (2004): Medien(sub)kulturen. Geschichte, Diskurse, Entwürfe. Bielefeld: transcript.

Jäckel, Michael (1999). Medienwirkungen. Ein Studienbuch zur Einführung. Opladen: Westdeutscher Verlag.

Janke, Klaus (1997). "Stars, Idole, Vorbilder. Was unterscheidet sie?« In: Schüler '97. Stars - Idole - Vorbilder. Hg. v. Christine Biermann und Klaus-Jürgen Tillmann. Seelze: Friedrich Verlag, S. 18-21.

Jenson, Joli (1992). »Fandom as Pathology: The Consequencens of Characterization. «In: The Adoring Audience. Fan Culture and Popular Media. Hg. v. Lisa A. Lewis. London, New York: Routledge, S. 9-29.

Lothwesen, Kai Stefan/Müllensiefen, Daniel (2004). „Vom Castingshow-Teilnehmer zum Star? Empirische Befunde zu den Urteilskategorien jugendlicher Musikhörer.« In: Diskussion Musikpädagogik, H. 23, S. 11-18.

Lowery, Shearon/DeFleur, Melvin L. (1995). Milestones in Mass Communication Research. Media Effects. White Plains, N.Y.: Longman (3. Aufl.).

Mayring, Philipp (1989). »Qualitative Inhaltsanalyse. In: Qualitative Forschung in der Psychologie: Grundlagen, Verfahrensweisen, Anwendungsfelder. Hg. v. Gerd Jüttemann. Heidelberg: Asanger, S. 187-211.

McCombs, Maxwell (2000). »Agenda Setting: Zusammenhänge zwischen Massenmedien und Weltbild.«In: Schorr 2000: 123-136.

Neckel, Sieghard (2004). »>Stars im Kumpelformat.< Der Soziologe Sieghard Neckel über Instant-Prominenz und das Risiko der Selbstdarstellung. «In: KulturSpiegel, H. 2 (Februar), S. 7-11.

Rhein, Stefanie (2002). »Bedeutungszuschreibungen an das eigene Musik-Fantum im Kontext aktueller Problembelastungen. Ergebnisse einer Befragung mit dem Multi-Media-Computer.«In: Wozu Jugendliche Musik und Medien gebrauchen. Jugendliche Identität und musikalische und mediale Geschmacksbildung. Hg. v. Renate Müller, Patrick Glogner, Stefanie Rhein und Jens Heim. München: Juventa, S. 43-56.

Rösing, Helmut/Barber-Kersovan, Alenka (1998). »Musikvermittlung in der modernen Mediengesellschaft. «In: Musikwissenschaft. Ein Grundkurs. Hg. v. Herbert Bruhn und Helmut Rösing. Reinbek: Rowohlt, S. 364-389.

Rubin, Alan (2000). "Die Uses-And-Gratifications-Perspektive der Medienwirkung. « In: Schorr 2000: 137-152. 
Schmiedke-Rindt, Carina (1992). »Express Yourself - Be Madonna With You.« Madonna-Fans und ihre Lebenswelt. Augsburg: Sonnentanz.

Schorr, Angela (Hg.) (2000). Publikums- und Wirkungsforschung. Ein Reader. Wiesbaden: Westdeutscher Verlag.

Six, Ulrike/Gleich, Ulrich (2000). "Sozio-emotionale und kognitive Reaktionen auf Ereignisszenarien mit TV-Personen. Ein Experiment zur parasozialen Beziehung.« In: Schorr 2000: 363-386.

Sommer, Carlo Michael (1997). "Stars als Mittel der Identitätskonstruktion. Überlegungen zum Phänomen des Star-Kults aus sozialpsychologischer Sicht.«In: Der Star. Geschichte - Rezeption - Bedeutung. Hg. v. Werner Faulstich und Helmut Korte. München: Fink, S. 114-124.

Vatterodt, Nikola (2000). Boygroups und ihre Fans. Annäherung an ein Popphänomen der neunziger Jahre. Karben: Coda.

Weyrauch, Jan (1997). Boygroups - Das Teenie-FANomen der 90er. Berlin: Extent.

Winter, Rainer (1997). "Medien und Fans. Zur Konstitution von Fan-Kulturen.« In: Kursbuch JugendKultur. Stile, Szenen und Identitäten vor der Jahrtausendwende. Hg. v. SpoKK. Mannheim: Bollmann, S. 40-53.

\begin{abstract}
In our survey we focus on young persons' categories of judgement when comparing well known German and international singers to contestants of German TV talent shows. Further questions concern the possible influence of the jury of Germany's most popular talent show (Deutschland sucht den Superstar) on the viewers' opinions and the fan community's musical interests and competence.

A questionnaire ascertained similarities between stars and contestants which were evaluated with the methods of multidimensional scaling and multidimensional unfolding. The fans' attitudes - as stated in contributions to internet discussion groups as well as judgements of the show's jury - were evaluated with the help of content analysis. The analysis reveals three major dimensions which display the young German TV-audience's categories of decision or judgement on music-»stars« in general. These categories are: casting vs. talent, crossmedia-coverage, nationality. The same categories were extracted from internet discussion groups. The sample showed that the consumers are well aware of musical details such as vocal abilities and professional skills. The meaning of stardom in general has changed due to the commercial success of TV talent shows.
\end{abstract}

